\title{
12. PERINATAL DEATHS
}

\section{Review of perinatal deaths 2004}

This chapter presents the results of perinatal death reviews carried out by the NSW Maternal and Perinatal Committee, which is a quality assurance committee established under the NSW Health Administration Act 1982. The Committee is privileged under the Act to carry out confidential reviews of maternal and perinatal deaths.

NSW Department of Health Policy Directive No. 2005_228 describes hospital procedures for review and reporting of perinatal deaths. The circular is available on the Department's website at www.health.nsw.gov. au/policies/PD/2005/PD2005_228.html. The Maternal and Perinatal Committee carries out reviews of perinatal deaths occurring among fetuses or infants of at least 22 weeks gestation or at least 500 grams birth weight. The criteria used by the NSW Midwives Data Collection (MDC) for reporting of births is at least 400 grams birth weight or at least 20 weeks gestation. The Maternal and Perinatal Committee reviews deaths that have a slightly higher threshold to focus attention on deaths that are more likely to be preventable.

Perinatal deaths were reviewed by the Committee's Perinatal Outcomes Working Party. Both stillbirths and neonatal deaths were classified according to an obstetric cause-specific classification, the Perinatal Society of Australia and New Zealand Perinatal Death Classification (PSANZ-PDC). Neonatal deaths were also classified by neonatal cause according to the Perinatal Society of Australia and New Zealand Neonatal Death Classification (PSANZ-NDC).

There were 649 perinatal deaths of at least 22 weeks gestation or at least 500 grams birth weight reported to the MDC in 2004. Confidential reports on 643 deaths were reviewed. Of the 471 stillbirths reported to the MDC, reviews were carried out on 449 (95.3 per cent). The MDC was notified of 178 neonatal deaths. However, reviews were carried out on 194 neonatal deaths, reflecting underenumeration of neonatal deaths on the MDC. Comparative information is also presented for 2001, 2002 and 2003.

\section{Trends in obstetric antecedents of perinatal death}

Between 2001 and 2004, the pattern of antecedent causes of death remained fairly stable (Table 143). Unexplained antepartum deaths comprised the largest category in 2004 (Figure 18), as for previous years. The next most common categories were fetal abnormalities followed by spontaneous preterm birth.

\section{Obstetric antecedents of perinatal death 2004}

\section{Congenital abnormality}

In 2004, congenital abnormalities were the underlying cause for 125 deaths (Table 144). The most common abnormalities were chromosomal ( $\mathrm{n}=35,28.0$ per cent). Of these, 14 were trisomy 18,8 were trisomy 21,5 were trisomy 13, 1 was Turner syndrome, and there were 7 other abnormalities.

Nineteen deaths were associated with abnormalities of the central nervous system (15.2 per cent), of which 8 were neural tube defects and a further 6 deaths were among babies with congenital hydrocephalus.

Twenty-four deaths were due to multiple abnormalities not associated with a chromosomal abnormality.

Twelve deaths were associated with abnormalities of the cardiovascular system, of which 4 were cases of hypoplastic left heart syndrome. Four deaths were associated with congenital diaphragmatic hernia.

\section{Perinatal infection}

Fourteen deaths were found to be due to infection, of which 6 were stillbirths and 8 were neonatal deaths. In 25 deaths there was an associated chorioamnionitis.

The most common infective organism identified was group B streptococcus, which was considered responsible for 3 stillbirths and 2 neonatal deaths. Two neonatal deaths were caused by Escherichia coli infection, one neonatal death following a Staphylococcus aureus infection, and one stillbirth following congenital cytomegalovirus infection. The causative organism was not specified for 5 deaths.

\section{Hypertension}

Thirty (23.4 per cent) deaths were considered to be due to maternal hypertension. There were 26 stillbirths and 4 neonatal deaths. The majority $(n=19,63.3$ per cent $)$ occurred in mothers with pre-eclampsia. Only one death in this group was associated with placental abruption, and one was associated with maternal diabetes.

\section{Antepartum haemorrhage}

Fifty-nine deaths were due to antepartum haemorrhage, of which 50 were due to placental abruption, one was due to placenta praevia, and 2 due to vasa praevia.

\section{Maternal disease}

Twenty-one deaths were attributed to other maternal conditions including: diabetes (8), maternal injury (2), termination of pregnancy (2), cholestasis (1), systemic lupus erythematosis (1), antiphospholipid syndrome (1), and maternal death (1). 


\begin{tabular}{|c|c|c|c|c|c|c|c|c|}
\hline \multirow[t]{3}{*}{ Obstetric antecedent } & \multicolumn{8}{|c|}{ Year } \\
\hline & \multicolumn{2}{|c|}{2001} & \multicolumn{2}{|c|}{2002} & \multicolumn{2}{|c|}{2003} & \multicolumn{2}{|c|}{2004} \\
\hline & No. & $\%$ & No. & $\%$ & No. & $\%$ & No. & $\%$ \\
\hline 1. Fetal abnormality & 90 & 14.6 & 103 & 16.8 & 95 & 16.0 & 125 & 19.4 \\
\hline 2. Perinatal infection & 28 & 4.5 & 27 & 4.4 & 30 & 5.0 & 14 & 2.2 \\
\hline 3. Hypertension & 41 & 6.7 & 34 & 5.5 & 32 & 5.4 & 30 & 4.7 \\
\hline 4. Antepartum haemorrhage & 48 & 7.8 & 52 & 8.5 & 44 & 7.4 & 59 & 9.2 \\
\hline 5. Maternal disease & 14 & 2.3 & 27 & 4.4 & 28 & 4.7 & 21 & 3.3 \\
\hline 6. Specific perinatal conditions & 52 & 8.4 & 45 & 7.3 & 51 & 8.6 & 43 & 6.7 \\
\hline 7. Hypoxic peripartum death & 22 & 3.6 & 23 & 3.8 & 21 & 3.5 & 20 & 3.1 \\
\hline 8. Fetal growth restriction & 5 & 0.8 & 13 & 2.1 & 10 & 1.7 & 16 & 2.5 \\
\hline 9. Spontaneous preterm & 120 & 19.5 & 126 & 20.6 & 94 & 15.8 & 121 & 18.8 \\
\hline 10. Unexplained antepartum death & 191 & 31.0 & 161 & 26.3 & 184 & 30.9 & 191 & 29.7 \\
\hline 11. No obstetric antecedent & 5 & 0.8 & 2 & 0.3 & 6 & 1.0 & 3 & 0.5 \\
\hline TOTAL & 616 & 100.0 & 613 & 100.0 & 595 & 100.0 & 643 & 100.0 \\
\hline
\end{tabular}

\section{FIGURE 18}

PERINATAL DEATHS BY OBSTETRIC ANTECEDENT AND YEAR, NSW 2004

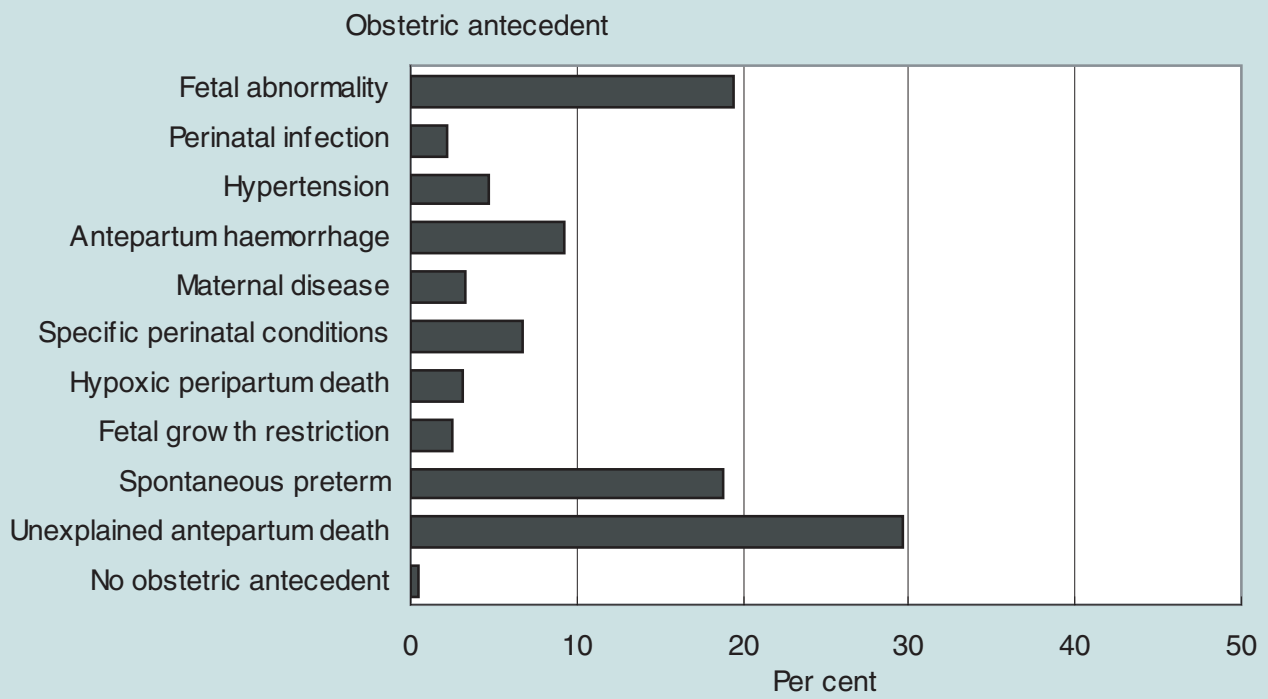

Source: NSW Maternal and Perinatal Committee, NSW Department of Health. 


\section{TABLE 144}

PERINATAL DEATHS BY OBSTETRIC ANTECEDENT AND PERINATAL OUTCOME, NSW 2004

\begin{tabular}{|c|c|c|c|c|c|c|c|}
\hline \multirow{3}{*}{\multicolumn{2}{|c|}{ Obstetric antecedent }} & \multirow{2}{*}{\multicolumn{2}{|c|}{ Stillbirth }} & \multicolumn{4}{|c|}{ Perinatal outcome } \\
\hline & & & & \multicolumn{2}{|c|}{ Neonatal death } & \multicolumn{2}{|c|}{ TOTAL } \\
\hline & & No. & $\%$ & No. & $\%$ & No. & $\%$ \\
\hline \multicolumn{8}{|c|}{ 1. Fetal abnormality } \\
\hline & Central nervous system & 10 & 2.2 & 9 & 4.6 & 19 & 3.0 \\
\hline & Cardiovascular system & 6 & 1.3 & 6 & 3.1 & 12 & 1.9 \\
\hline & Urinary tract & 3 & 0.7 & 4 & 2.1 & 7 & 1.1 \\
\hline & Gastrointestinal system & 1 & 0.2 & 1 & 0.5 & 2 & 0.3 \\
\hline & Chromosomal & 21 & 4.7 & 14 & 7.2 & 35 & 5.4 \\
\hline & Metabolic & 1 & 0.2 & 3 & 1.5 & 4 & 0.6 \\
\hline & Multiple & 7 & 1.6 & 17 & 8.8 & 24 & 3.7 \\
\hline & Other & 10 & 2.2 & 11 & 5.7 & 21 & 3.3 \\
\hline & Unspecified & 1 & 0.2 & 0 & 0.0 & 1 & 0.2 \\
\hline & Total & 60 & 13.4 & 65 & 33.5 & 125 & 19.4 \\
\hline \multicolumn{8}{|c|}{ 2. Perinatal infection } \\
\hline & Group B Streptococcus & 3 & 0.7 & 2 & 1.0 & 5 & 0.8 \\
\hline & E. coli & 0 & 0.0 & 2 & 1.0 & 2 & 0.3 \\
\hline & Other bacterial & 0 & 0.0 & 1 & 0.5 & 1 & 0.2 \\
\hline & Unspecified bacterial & 2 & 0.4 & 1 & 0.5 & 3 & 0.5 \\
\hline & Cytomegalovirus & 1 & 0.2 & 0 & 0.0 & 1 & 0.2 \\
\hline & Unspecified organism & 0 & 0.0 & 2 & 1.0 & 2 & 0.3 \\
\hline & Total & 6 & 1.3 & 8 & 4.1 & 14 & 2.2 \\
\hline \multicolumn{8}{|c|}{ 3. Hypertension } \\
\hline & Chronic: Essential & 2 & 0.4 & 0 & 0.0 & 2 & 0.3 \\
\hline & Chronic: Secondary (for example, renal) & 2 & 0.4 & 0 & 0.0 & 2 & 0.3 \\
\hline & Gestational & 3 & 0.7 & 0 & 0.0 & 3 & 0.5 \\
\hline & Pre-eclampsia & 14 & 3.1 & 3 & 1.5 & 17 & 2.6 \\
\hline & Pre-eclampsia superimposed on & & & & & & \\
\hline & pre-existing hypertension & 1 & 0.2 & 1 & 0.5 & 2 & 0.3 \\
\hline & Unspecified & 4 & 0.9 & 0 & 0.0 & 4 & 0.6 \\
\hline & Total & 26 & 5.8 & 4 & 2.1 & 30 & 4.7 \\
\hline \multicolumn{8}{|c|}{ 4. Antepartum haemorrhage } \\
\hline & Placental abruption & 36 & 8.0 & 14 & 7.2 & 50 & 7.8 \\
\hline & Placenta praevia & 1 & 0.2 & 0 & 0.0 & 1 & 0.2 \\
\hline & Vasa praevia & 2 & 0.4 & 0 & 0.0 & 2 & 0.3 \\
\hline & Undetermined origin & 5 & 1.1 & 1 & 0.5 & 6 & 0.9 \\
\hline & Total & 44 & 9.8 & 15 & 7.7 & 59 & 9.2 \\
\hline \multicolumn{8}{|c|}{ 5. Maternal disease } \\
\hline \multicolumn{8}{|c|}{ Termination of pregnancy } \\
\hline & other than for fetal abnormality & 1 & 0.2 & 1 & 0.5 & 2 & 0.3 \\
\hline & Diabetes-gestational diabetes & 8 & 1.8 & 0 & 0.0 & 8 & 1.2 \\
\hline & Maternal injury: Accidental & 1 & 0.2 & 1 & 0.5 & 2 & 0.3 \\
\hline & Other & 8 & 1.8 & 1 & 0.5 & 9 & 1.4 \\
\hline & Total & 18 & 4.0 & 3 & 1.5 & 21 & 3.3 \\
\hline \multicolumn{8}{|c|}{ 6. Specific perinatal conditions } \\
\hline & Twin-to-twin transfusion & 14 & 3.1 & 7 & 3.6 & 21 & 3.3 \\
\hline & Fetomaternal haemorrhage & 4 & 0.9 & 0 & 0.0 & 4 & 0.6 \\
\hline & Antepartum cord complications & 6 & 1.3 & 0 & 0.0 & 6 & 0.9 \\
\hline & Uterine abnormality & 0 & 0.0 & 1 & 0.5 & 1 & 0.2 \\
\hline & Haemolytic disease & 1 & 0.2 & 0 & 0.0 & 1 & 0.2 \\
\hline & Idiopathic hydrops & 5 & 1.1 & 4 & 2.1 & 9 & 1.4 \\
\hline & Other & 1 & 0.2 & 0 & 0.0 & 1 & 0.2 \\
\hline & Total & 31 & 6.9 & 12 & 6.2 & 43 & 6.7 \\
\hline 7. & Hypoxic peripartum death & & & & & & \\
\hline & Uterine rupture & 1 & 0.2 & 2 & 1.0 & 3 & 0.5 \\
\hline & Cord prolapse & 2 & 0.4 & 0 & 0.0 & 2 & 0.3 \\
\hline & Shoulder dystocia & 0 & 0.0 & 1 & 0.5 & 1 & 0.2 \\
\hline & Other intrapartum complication & 3 & 0.7 & 6 & 3.1 & 9 & 1.4 \\
\hline & No intrapartum complication & 1 & 0.2 & 1 & 0.5 & 2 & 0.3 \\
\hline & Unspecified & 2 & 0.4 & 1 & 0.5 & 3 & 0.5 \\
\hline & Total & 9 & 2.0 & 11 & 5.7 & 20 & 3.1 \\
\hline 8. & Fetal growth restriction & & & & & & \\
\hline & With evidence of uteroplacental insufficiency & 6 & 1.3 & 2 & 1.0 & 8 & 1.2 \\
\hline & With chronic villitis & 0 & 0.0 & 2 & 1.0 & 2 & 0.3 \\
\hline & Without the above placental pathology & 2 & 0.4 & 2 & 1.0 & 4 & 0.6 \\
\hline & No placental examination & 0 & 0.0 & 2 & 1.0 & 2 & 0.3 \\
\hline & Total & 8 & 1.8 & 8 & 4.1 & 16 & 2.5 \\
\hline
\end{tabular}




\section{TABLE 144 (continued)}

\section{PERINATAL DEATHS BY OBSTETRIC ANTECEDENT AND PERINATAL OUTCOME, NSW 2004}

\begin{tabular}{|c|c|c|c|c|c|c|}
\hline \multirow[t]{3}{*}{ Obstetric antecedent } & \multicolumn{6}{|c|}{ Perinatal outcome } \\
\hline & \multicolumn{2}{|c|}{ Stillbirth } & \multicolumn{2}{|c|}{ Neonatal death } & \multicolumn{2}{|c|}{ TOTAL } \\
\hline & No. & $\%$ & No. & $\%$ & No. & $\%$ \\
\hline \multicolumn{7}{|l|}{$\begin{array}{l}\text { 9. Spontaneous preterm } \\
\text { Intact membranes or membrane rupture } \\
\text { less than } 24 \text { hours: }\end{array}$} \\
\hline with chorioamnionitis & 12 & 2.7 & 21 & 10.8 & 33 & 5.1 \\
\hline without chorioamnionitis & 9 & 2.0 & 16 & 8.2 & 25 & 3.9 \\
\hline no placental examination & 2 & 0.4 & 4 & 2.1 & 6 & 0.9 \\
\hline unspecified placental examination & 3 & 0.7 & 0 & 0.0 & 3 & 0.5 \\
\hline Membrane rupture 24 hours or more: & & & & & & \\
\hline with chorioamnionitis & 18 & 4.0 & 15 & 7.7 & 33 & 5.1 \\
\hline without chorioamnionitis & 3 & 0.7 & 5 & 2.6 & 8 & 1.2 \\
\hline no placental examination & 0 & 0.0 & 1 & 0.5 & 1 & 0.2 \\
\hline Membrane rupture unknown duration: & & & & & & \\
\hline with chorioamnionitis & 4 & 0.9 & 0 & 0.0 & 4 & 0.6 \\
\hline without chorioamnionitis & 2 & 0.4 & 2 & 1.0 & 4 & 0.6 \\
\hline no placental examination & 3 & 0.7 & 1 & 0.5 & 4 & 0.6 \\
\hline Total & 56 & 12.5 & 65 & 33.5 & 121 & 18.8 \\
\hline \multicolumn{7}{|l|}{ 10. Unexplained antepartum death } \\
\hline With evidence of uteroplacental insufficiency & 40 & 8.9 & 0 & 0.0 & 40 & 6.2 \\
\hline With chronic villitis & 5 & 1.1 & 0 & 0.0 & 5 & 0.8 \\
\hline Without the above placental pathology & 127 & 28.3 & 0 & 0.0 & 127 & 19.8 \\
\hline No placental examination & 14 & 3.1 & 0 & 0.0 & 14 & 2.2 \\
\hline Unspecified placental examination & 5 & 1.1 & 0 & 0.0 & 5 & 0.8 \\
\hline Total & 191 & 42.5 & 0 & 0.0 & 191 & 29.7 \\
\hline 11. No obstetric antecedent & 0 & 0.0 & 3 & 1.5 & 3 & 0.5 \\
\hline TOTAL & 100.0 & 194 & 100.0 & 643 & 100.0 & \\
\hline
\end{tabular}

\section{Specific perinatal conditions}

Of the 43 deaths in this group, twin-to-twin transfusion accounted for 21 deaths, followed by idiopathic hydrops (9), antepartum cord complications (6) and fetomaternal haemorrhage (4). Other causes were: uterine abormality (1), haemolytic disease (1), and maternal autoimmune disease (1).

\section{Hypoxic peripartum death}

There were 20 deaths associated with peripartum hypoxia. Three deaths followed uterine rupture. Two deaths followed cord prolapse and one death followed shoulder dystocia.

One death occurred before the onset of labour, 4 during labour and 4 at an unspecified time prior to birth. The remaining 11 deaths occurred in the neonatal period.

\section{Fetal growth restriction}

In 16 cases, the main obstetric cause of death was considered to be fetal growth restriction (FGR). Of these, 8 were stillbirths and 8 were neonatal deaths.

FGR is defined as less than the tenth percentile of birth weight for gestational age with no major congenital abnormalities. If a maternal or fetal cause of FGR was known then the cause of death was classified to the underlying cause of the FGR. Stillbirths with evidence of maceration were not classified as FGR unless there was evidence of growth restriction on serial ultrasound during pregnancy.

\section{Spontaneous preterm}

There were 121 perinatal deaths associated with spontaneous preterm birth, which comprises normally formed and appropriately grown babies born before 37 weeks gestation. Of these, 56 (46.3 per cent) were stillbirths and 65 (53.7 per cent) were neonatal deaths.

Forty-four deaths (36.4 per cent) were at less than 23 weeks gestation, 59 ( 48.8 per cent) were at 23-25 weeks gestation, and 18 (14.9 per cent) occurred between 26 and 36 weeks gestation. Thirty-six deaths ( 29.8 per cent) were associated with membrane rupture of 24 hours or more.

\section{Unexplained antepartum death}

Of the 191 unexplained stillbirths, 122 (63.9 per cent) were low birth weight babies and 114 (59.7 per cent) were premature. A variety of associated maternal conditions were reported in this group including: multiple pregnancy (9 deaths), maternal hypertension (11), diabetes (5), epilepsy (2), asthma (2), systemic lupus erythematosis (2), drug abuse (1) and cholestasis of pregnancy (1). Placental histopathology results were provided for 176 unexplained antepartum deaths (92.1 per cent) and evidence of uteroplacental insufficiency was found in 40 .

\section{No obstetric antecedent}

No obstetric cause of death could be identified for 3 neonatal deaths. Two of these babies had a post-mortem examination. Placental histopathology examination had been carried out in 2 cases. 


\section{Obstetric cause of perinatal death by hospital service level 2004}

Obstetric service levels are described in the Explanatory Notes of the Methods section (page 16). The majority of perinatal deaths occurred in level 6 hospitals $(52.7$ per cent, Table 145). The proportion of unexplained intrauterine deaths was substantially lower in level 6 hospitals than other hospitals, possibly due to better access to perinatal post-mortem services. The proportion of deaths associated with congenital abnormalities was highest in level 6 hospitals, reflecting patterns of referral for diagnosis and treatment.

\section{Time of death 2004}

Of the 643 perinatal deaths in 2004, 292 (45.4 per cent) occurred before the onset of labour, 46 ( 7.2 per cent) occurred during labour, 111 (17.3 per cent) occurred at an unknown time before birth, and 194 (30.3 per cent) were neonatal deaths.

\section{Trends in neonatal causes of death}

Between 2001 and 2004 extreme prematurity was the most common cause of neonatal death, accounting for over one-third of all neonatal deaths in 2004 (Table 146). Congenital abnormalities were the next most common cause of neonatal death for the 4 years. There were slightly more deaths attributed to congenital abnormalities in 2004 compared with previous years.

\section{Neonatal causes of death 2004}

Of the 194 neonatal deaths reviewed for 2004, 154 (79.4 per cent) were less than 37 weeks gestation (Table 147). The most common neonatal cause of death was extreme prematurity ( $n=67,34.5$ per cent). Fifty-six infants died from a congenital abnormality. There were 20 deaths due to hypoxic ischaemic encephalopathy and 12 deaths due to intracranial haemorrhage.

\section{Perinatal deaths associated with maternal drug dependency-abuse 2004}

No perinatal deaths were directly attributed to maternal drug dependency or drug abuse. Eleven deaths occurred among mothers who had a history of drug dependency or abuse, but drug use was not considered to be the main cause of death.

\section{Post-mortem examination 2004}

Postmortem examination is valuable in ascertaining or confirming the cause of death, identifying additional factors that may have contributed to the death, and counselling parents about the cause of death. Postmortem examinations were carried out for 212 (33.0 per cent) deaths: 166 stillborn infants (37.0 per cent of all reported stillbirths) and 46 neonatal deaths (23.7 per cent of all reported neonatal deaths). Placental histopathology was carried out in 543 perinatal deaths ( 84.5 per cent).

\section{TABLE 145}

PERINATAL DEATHS BY OBSTETRIC ANTECEDENT AND HOSPITAL SERVICE LEVEL, NSW 2004

\begin{tabular}{|c|c|c|c|c|c|c|c|c|c|c|c|c|c|c|}
\hline \multirow[t]{3}{*}{ Obstetric antecedent } & \multirow{2}{*}{\multicolumn{2}{|c|}{ Level 2}} & \multirow{2}{*}{\multicolumn{2}{|c|}{ Level 3}} & \multicolumn{4}{|c|}{ Hospital service level } & \multirow{2}{*}{\multicolumn{2}{|c|}{ Level 6}} & \multirow{2}{*}{\multicolumn{2}{|c|}{ Private }} & \multirow{2}{*}{\multicolumn{2}{|c|}{ TOTAL }} \\
\hline & & & & & & vel 4 & & vel 5 & & & & & & \\
\hline & No. & $\%$ & No. & $\%$ & No. & $\%$ & No. & $\%$ & No. & $\%$ & No. & $\%$ & No. & $\%$ \\
\hline 1. Fetal abnoramality & 0 & 0.0 & 6 & 14.6 & 9 & 9.2 & 11 & 12.6 & 93 & 27.4 & 6 & 8.7 & 125 & 19.4 \\
\hline 2. Perinatal infection & 0 & 0.0 & 0 & 0.0 & 2 & 2.0 & 1 & 1.1 & 9 & 2.7 & 2 & 2.9 & 14 & 2.2 \\
\hline 3. Hypertension & 0 & 0.0 & 1 & 2.4 & 6 & 6.1 & 6 & 6.9 & 13 & 3.8 & 4 & 5.8 & 30 & 4.7 \\
\hline 4. Antepartum haemorrhage & 2 & 28.6 & 4 & 9.8 & 11 & 11.2 & 9 & 10.3 & 29 & 8.6 & 4 & 5.8 & 59 & 9.2 \\
\hline 5. Maternal disease & 0 & 0.0 & 2 & 4.9 & 5 & 5.1 & 2 & 2.3 & 10 & 2.9 & 2 & 2.9 & 21 & 3.3 \\
\hline 6. Specific perinatal conditions & 0 & 0.0 & 2 & 4.9 & 3 & 3.1 & 7 & 8.0 & 24 & 7.1 & $\overline{7}$ & 10.1 & 43 & 6.7 \\
\hline 7. Hypoxic peripartum death & 2 & 28.6 & 2 & 4.9 & 0 & 0.0 & 2 & 2.3 & 13 & 3.8 & 1 & 1.4 & 20 & 3.1 \\
\hline 8. Fetal growth restriction" & 0.0 & 0 & 0.0 & 3 & 3.1 & 1 & 1.1 & 12 & 3.5 & 0 & 0.0 & 16 & 2.5 & \\
\hline 9. Spontaneous preterm & 0 & 0.0 & 8 & 19.5 & 13 & 13.3 & 13 & 14.9 & 74 & 21.8 & 12 & 17.4 & 121 & 18.8 \\
\hline 10. Unexplained antepartum death & 3 & 42.9 & 15 & 36.6 & 46 & 46.9 & 35 & 40.2 & 61 & 18.0 & 31 & 44.9 & 191 & 29.7 \\
\hline 11. No obstetric antecedent & 0 & 0.0 & 1 & 2.4 & 0 & 0.0 & 0 & 0.0 & 1 & 0.3 & 0 & 0.0 & 3 & 0.5 \\
\hline TOTAL & 7 & 100.0 & 41 & 100.0 & 98 & 100.0 & 87 & 100.0 & 339 & 100.0 & 69 & 100.0 & 643 & 100.0 \\
\hline
\end{tabular}

Source: NSW Maternal and Perinatal Committee, NSW Department of Health.

\# The spontaneous preterm and no obstetric antecedent categories each include one perinatal death that occurred in a level 1 hospital. 


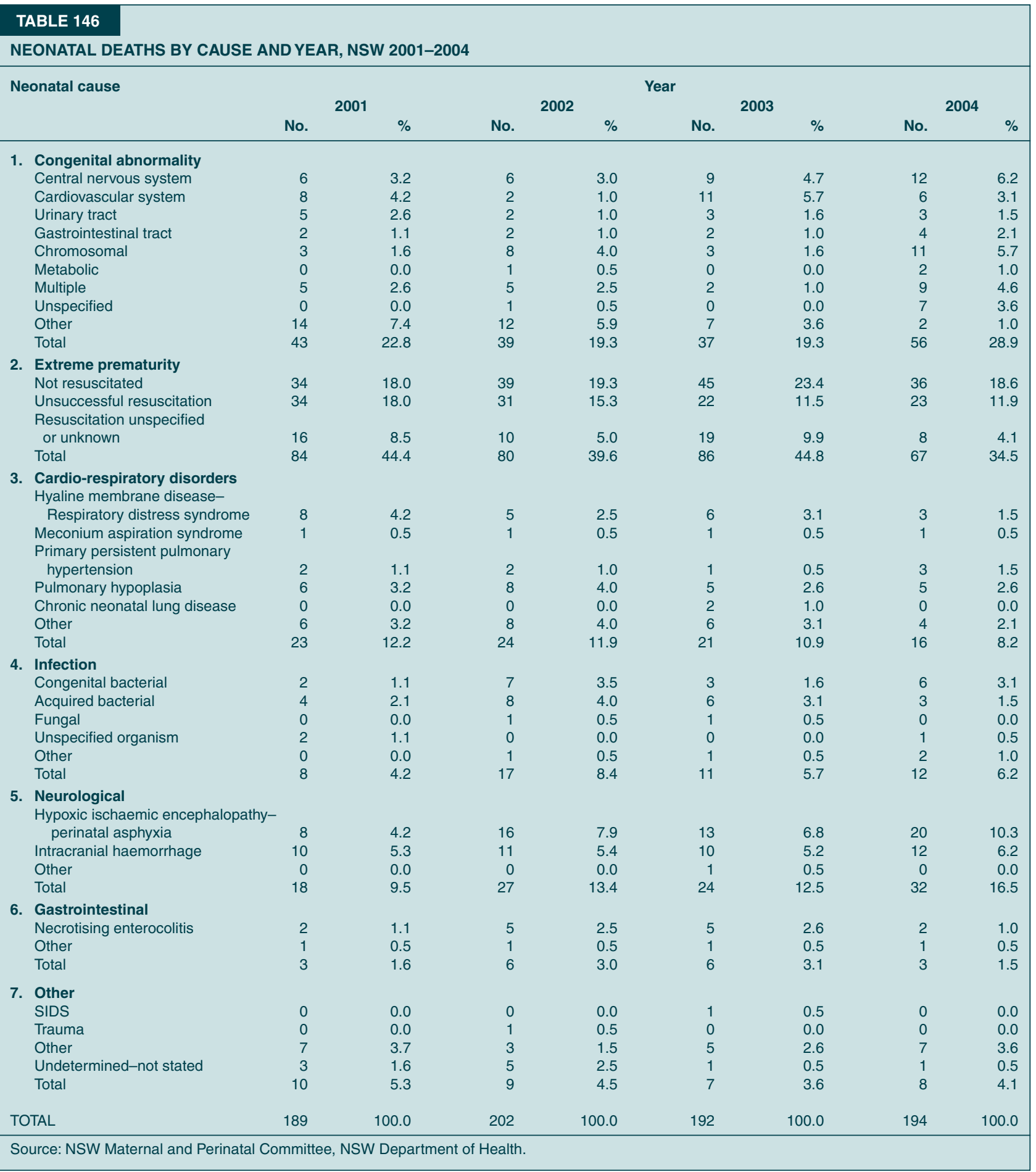




\section{TABLE 147}

NEONATAL DEATHS BY CAUSE AND GESTATIONAL AGE, NSW 2004

Neonatal cause

Gestational age (weeks)

\begin{tabular}{|c|c|c|c|c|c|c|}
\hline & \multicolumn{2}{|c|}{ Less than 37} & & TOTAL \\
\hline & No. & $\%$ & No. & $\%$ & No. & $\%$ \\
\hline 1. Congenital abnormality & & & & & & \\
\hline Central nervous system & 9 & 5.8 & 3 & 7.5 & 12 & 6.2 \\
\hline Cardiovascular system & 2 & 1.3 & 4 & 10.0 & 6 & 3 \\
\hline Urinary tract & 3 & 1.9 & 0 & 0.0 & 3 & 1.5 \\
\hline Gastrointestinal tract & 3 & 1.9 & 1 & 2.5 & 4 & \\
\hline Chromosomal & 9 & 5.8 & 2 & 5.0 & 11 & 5.7 \\
\hline Metabolic & 0 & 0.0 & 2 & 5.0 & 2 & 1.0 \\
\hline Multiple & 7 & 4.5 & 2 & 5.0 & 9 & 4 \\
\hline Other & 5 & 3.2 & 2 & 5.0 & 7 & 3 \\
\hline Unspecified & 0 & 0.0 & 2 & 5.0 & 2 & 1 \\
\hline Total & 38 & 24.7 & 18 & 45.0 & 56 & 28.9 \\
\hline 2. Extreme prematurity & & & & & & \\
\hline Not resuscitated & 36 & 23.4 & 0 & 0.0 & 36 & 18.6 \\
\hline Unsuccessful resuscitation & 23 & 14.9 & 0 & 0.0 & 23 & 11.9 \\
\hline Resuscitation unspecified or unknown & 8 & 5.2 & 0 & 0.0 & 8 & \\
\hline Total & 67 & 43.5 & 0 & 0.0 & 67 & 34 \\
\hline
\end{tabular}

3. Cardio-respiratory disorders

Hyaline membrane disease

Respiratory distress syndrome

Meconium aspiration syndrome

Primary persistent pulmonary hypertension

Pulmonary hypoplasia

Other

Total

4. Infection

Congenital bacterial

Acquired bacterial

Unspecified organism

Other

Total

$\%$

5. Neurological

Hypoxic ischaemic encephalopathy-

perinatal asphyxia

Intracranial haemorrhage

Total

13
0

6. Gastrointestinal

Other

Necrotising enterocolitis

Total

7. Other
Undetermined-Unknown
Other
Total

$\begin{array}{ll}0 & 0.0 \\ 7 & 4.5\end{array}$

0.0
4.5
4.5

0

0.0

$\begin{array}{ll}0 & 0.0 \\ 0 & 0.0\end{array}$

$1 \quad 0.5$

1.9

1.0

TOTAL

154

100.0

$\begin{array}{ll}1 & 2.5 \\ 0 & 0.0 \\ 1 & 2.5\end{array}$

2.5
0.0
2.5

0.5

4.1

Source: NSW Maternal and Perinatal Committee, NSW Department of Health 